\title{
Adenine-based $\mathrm{Zn}(\mathrm{II}) / \mathrm{Cd}(\mathrm{II})$ Metal-organic Frameworks as Efficient Heterogeneous Catalysts for Facile $\mathrm{CO}_{2}$ Fixation into Cyclic Carbonates: $\mathrm{A}$ DFT Supported Study of the Reaction Mechanism
}

Yadagiri Rachuri, ${ }^{a}$ Jintu Francis Kurisingal, ${ }^{a}$ Ramesh Kumar Chitumalla, ${ }^{b}$ Srimai Vuppala, ${ }^{b}$

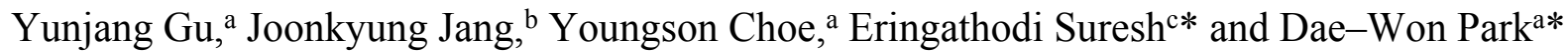

${ }^{a}$ Division of Chemical and Biomolecular Engineering, Pusan National University, Busan 46241, Korea.

${ }^{b}$ Department of Nanoenergy Engineering, Pusan National University, Busan 46241, Republic of Korea.

${ }^{c}$ Analytical and Environmental Science Division and Centralized Instrument Facility, CSIRCentral Salt and Marine Chemicals Research Institute, G. B. Marg, Bhavnagar-364 002, India.

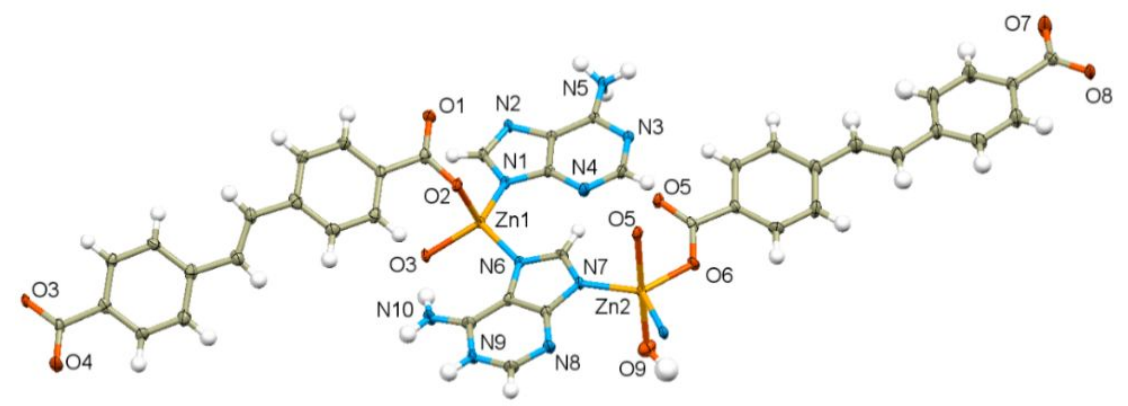

Figure S1. ORTEP diagram depicting coordination environment in PNU-21.

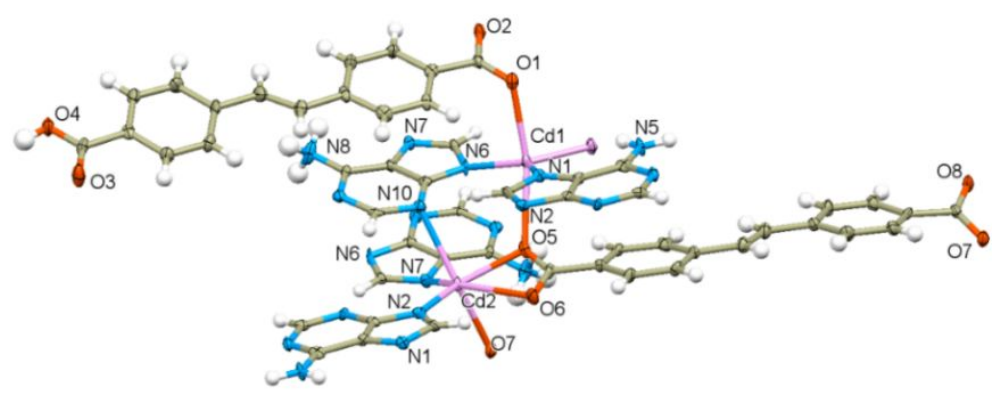

Figure S2. ORTEP diagram depicting coordination environment in PNU-22. 


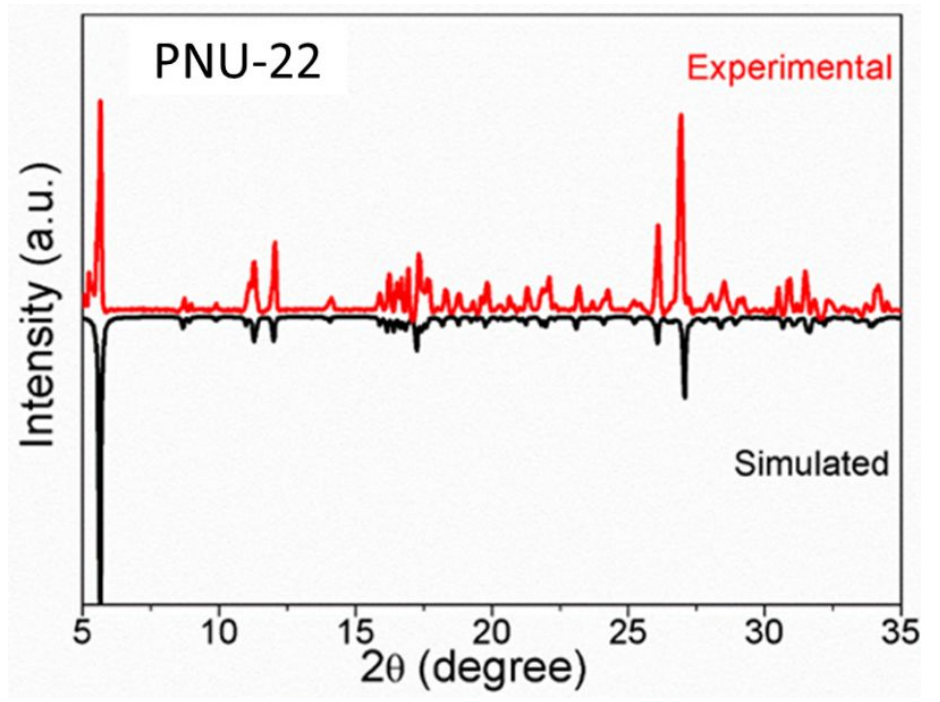

Figure S3. Simulated and experimental PXRD patterns of PNU-22.

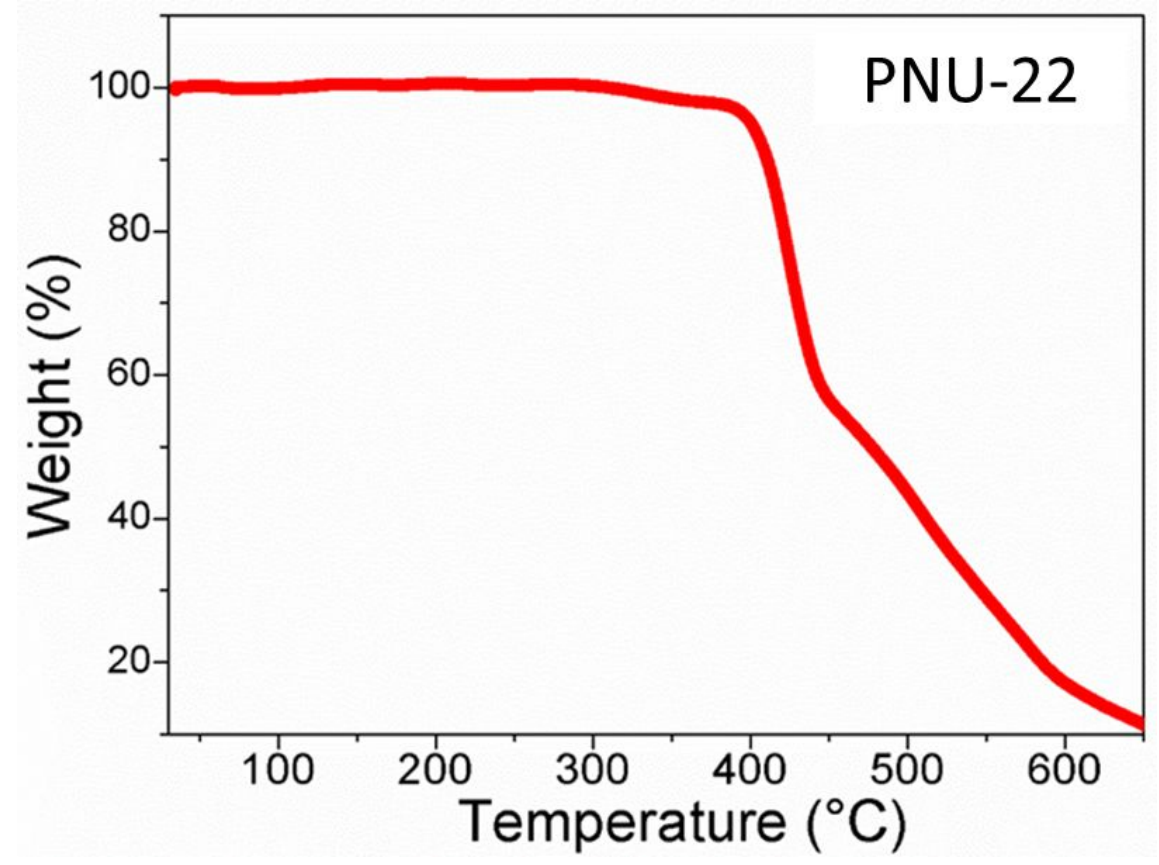

Figure S4. TGA profile of PNU-22. 


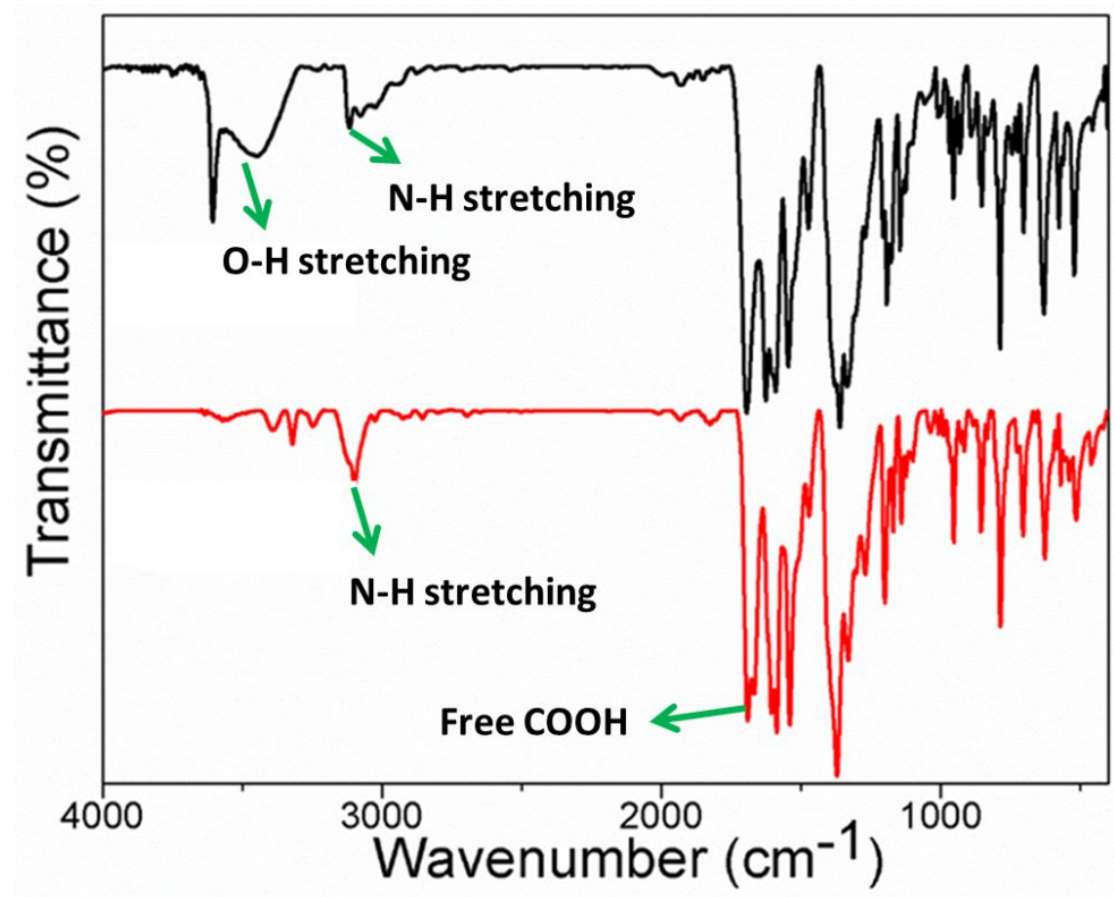

Figure S5. FT-IR spectra of PNU-21 and PNU-22.
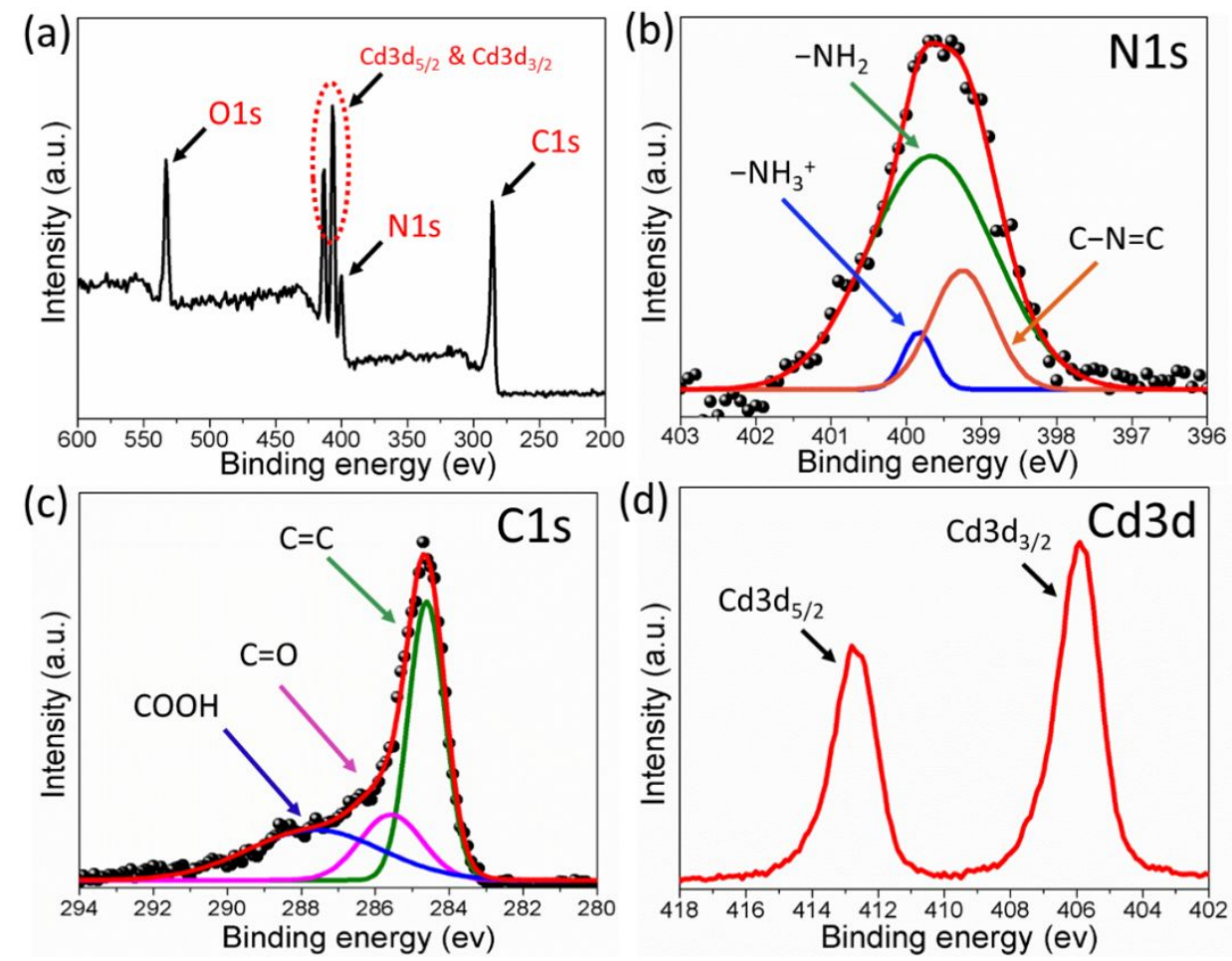

Figure S6. XPS spectra of PNU-22. (a) Full spectrum, (b) N1s, (c) C1s and (d) Cd3d. 


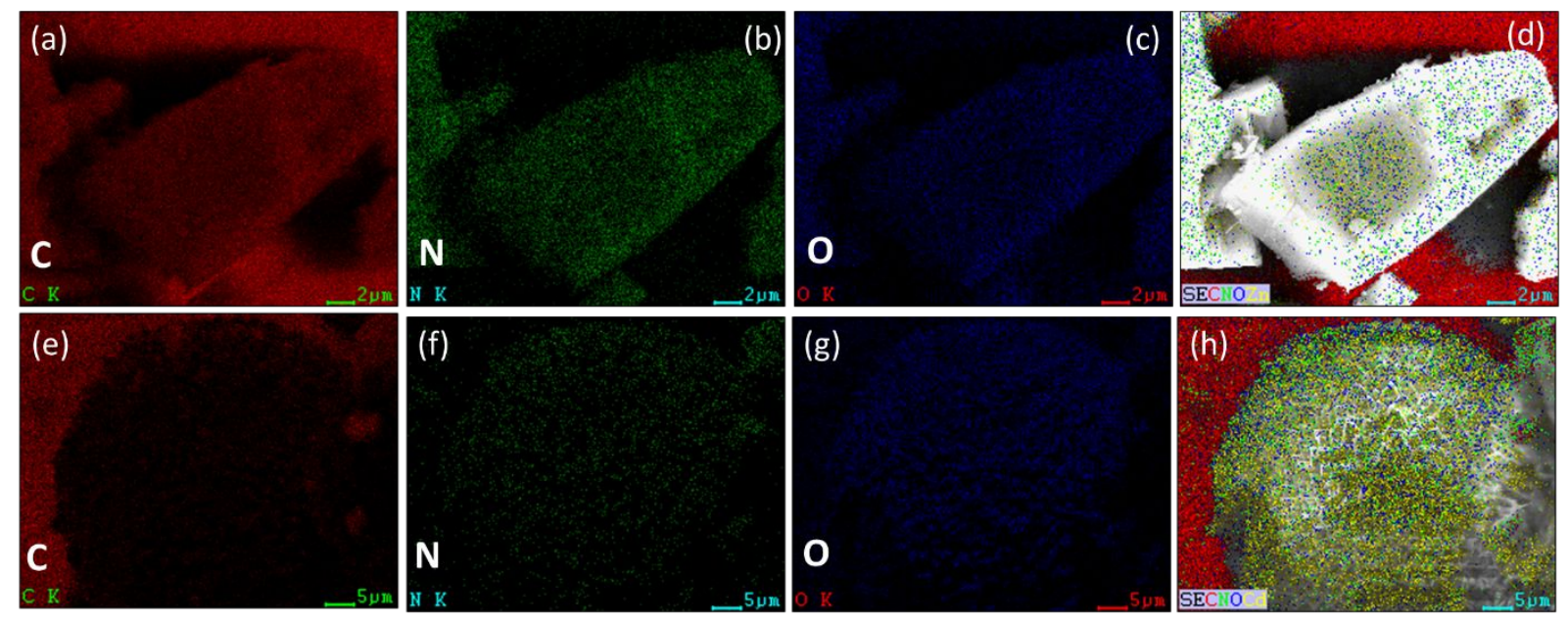

Figure S7. Elemental mapping of each element (C, N, O) in PNU-21 (a-d) and PNU-22(e-h).

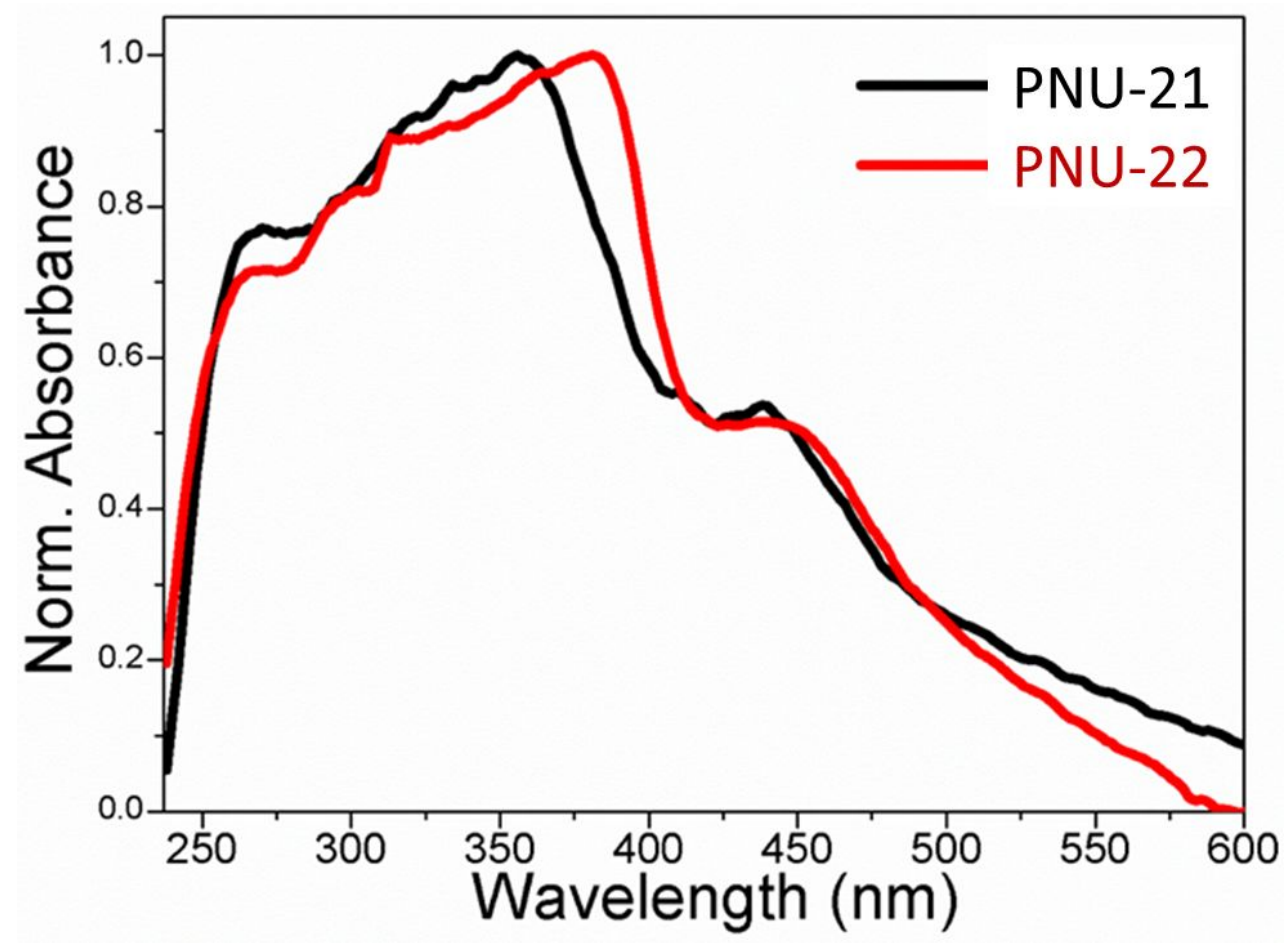

Figure S8. Absorbance spectrum of PNU-21 and PNU-22. 

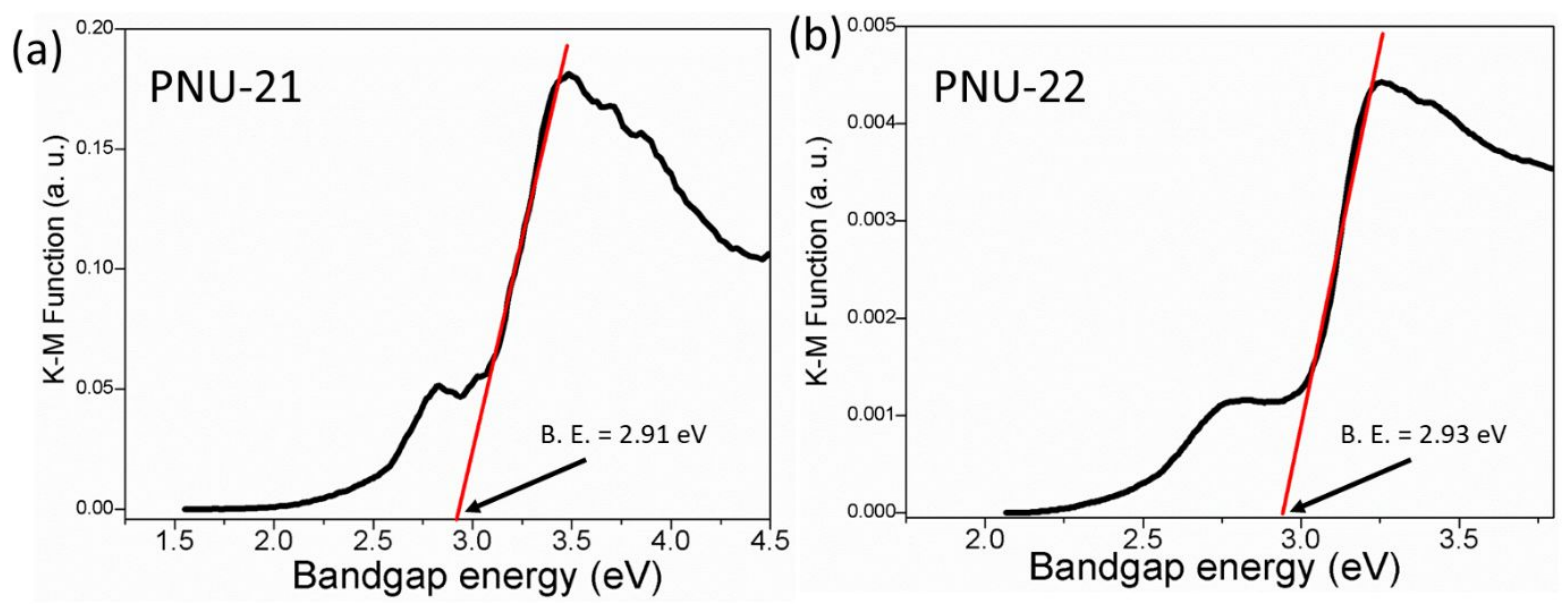

Figure S9. (a-b) Band gap energies were calculated by K-M method for PNU-21 and PNU-22.

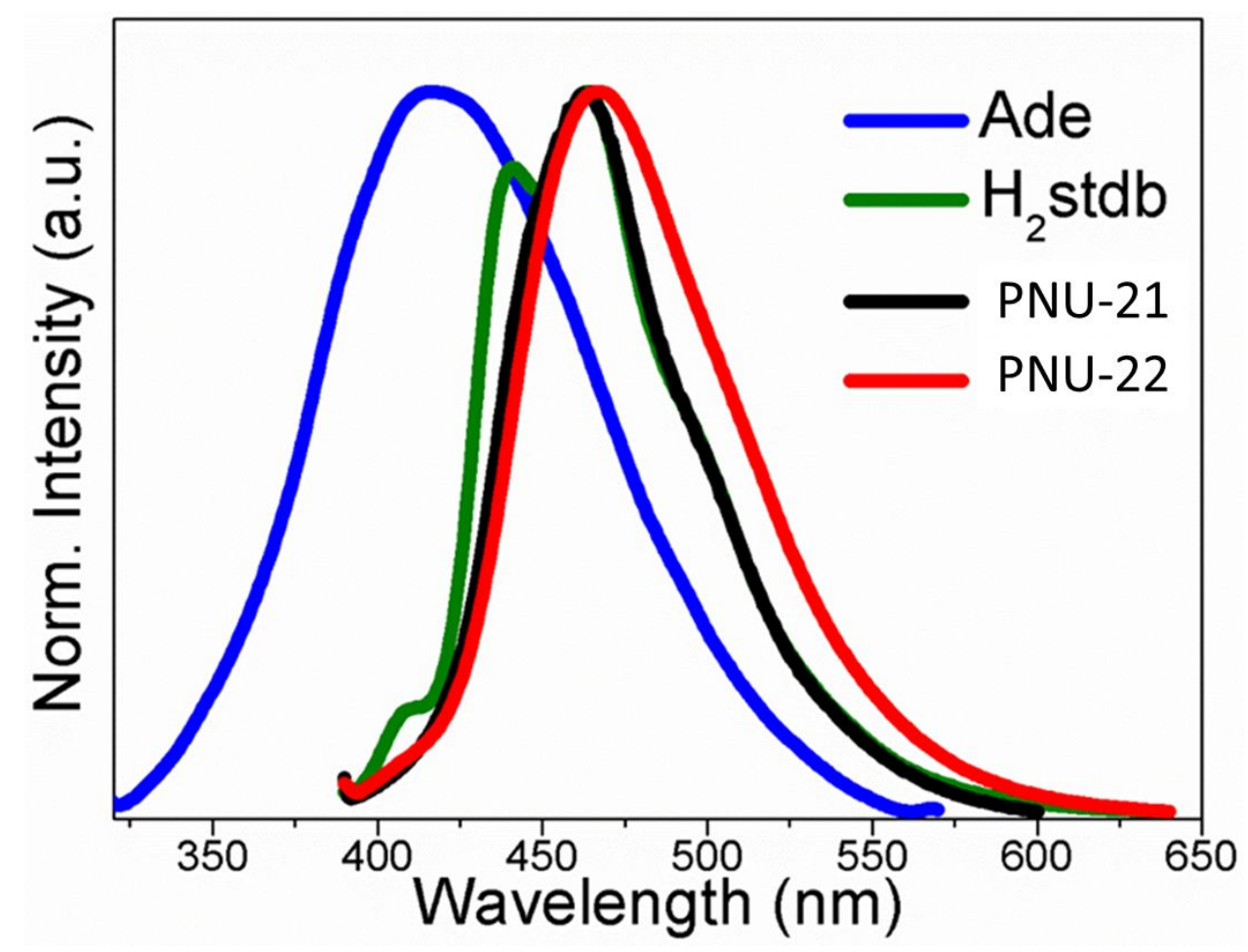

Figure S10. Fluorescence spectra of Ade, stdb, PNU-21 and PNU-22 (Ade, $\lambda_{\mathrm{em}}=416 \mathrm{~nm}, \lambda_{\mathrm{ex}}=$ $295 \mathrm{~nm}$; stdb, $\lambda_{\mathrm{em}}=463 \mathrm{~nm}, \lambda_{\mathrm{ex}}=330 \mathrm{~nm}$; PNU-21 and $2, \lambda_{\mathrm{em}}=465$ and $\left.467 \mathrm{~nm}, \lambda_{\mathrm{ex}}=380 \mathrm{~nm}\right)$. 

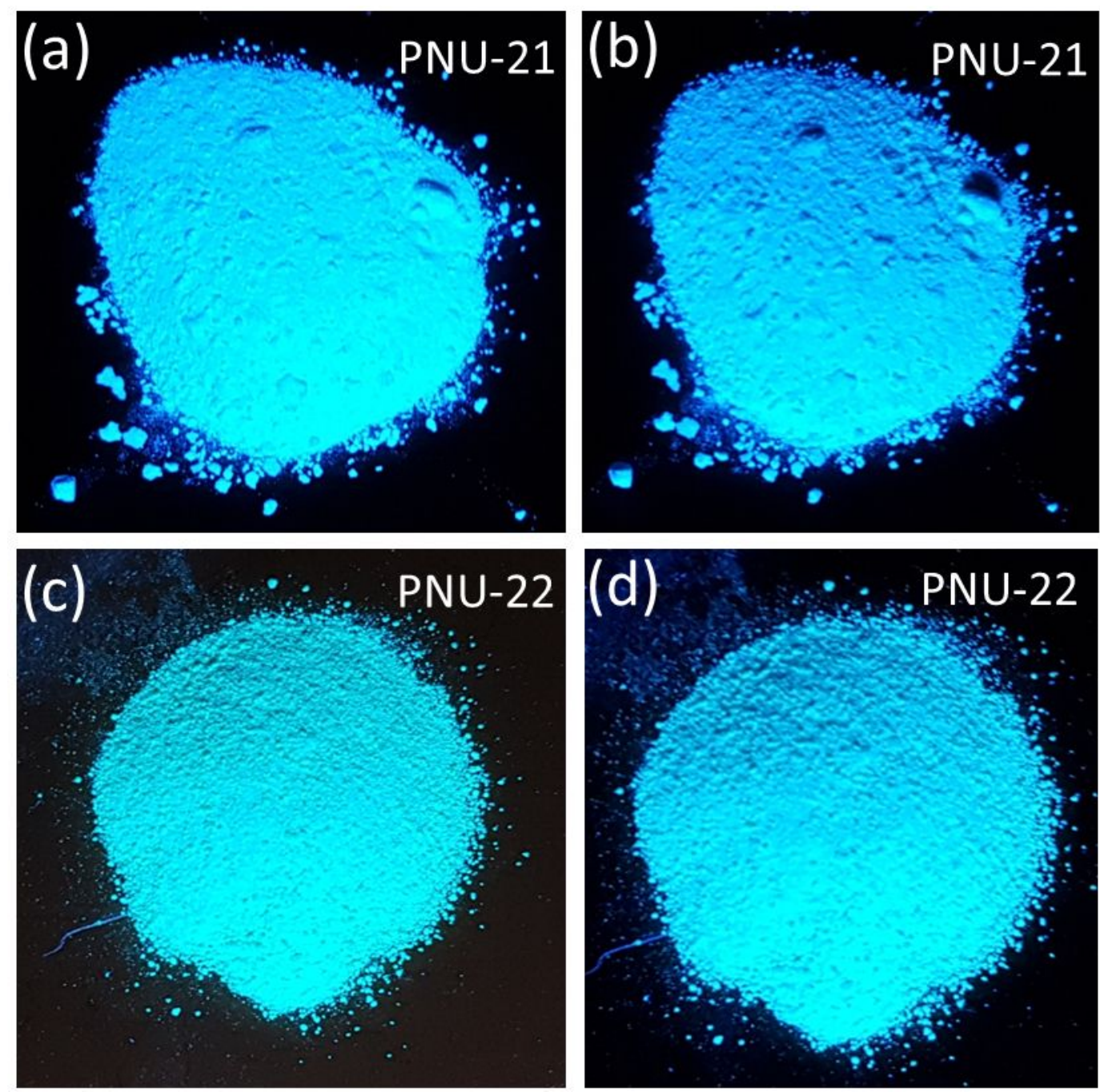

Figure S11. Digital images of PNU-21 and PNU-22 under UV light at $250(\mathrm{a}, \mathrm{c})$ and $365 \mathrm{~nm}$ $(b, d)$.
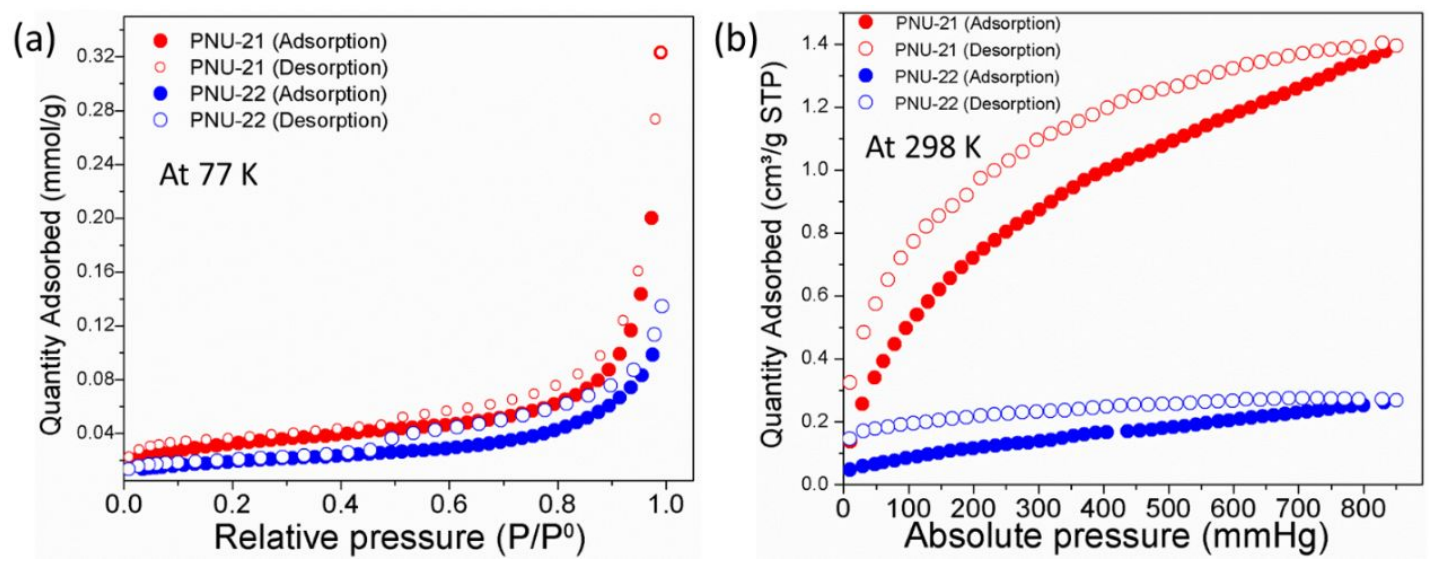

Figure S12. (a) $\mathrm{N}_{2}$ and (b) $\mathrm{CO}_{2}$ adsorption-desorption isotherms of prepared catalysts: PNU-21 (red) and PNU-22 (blue). 

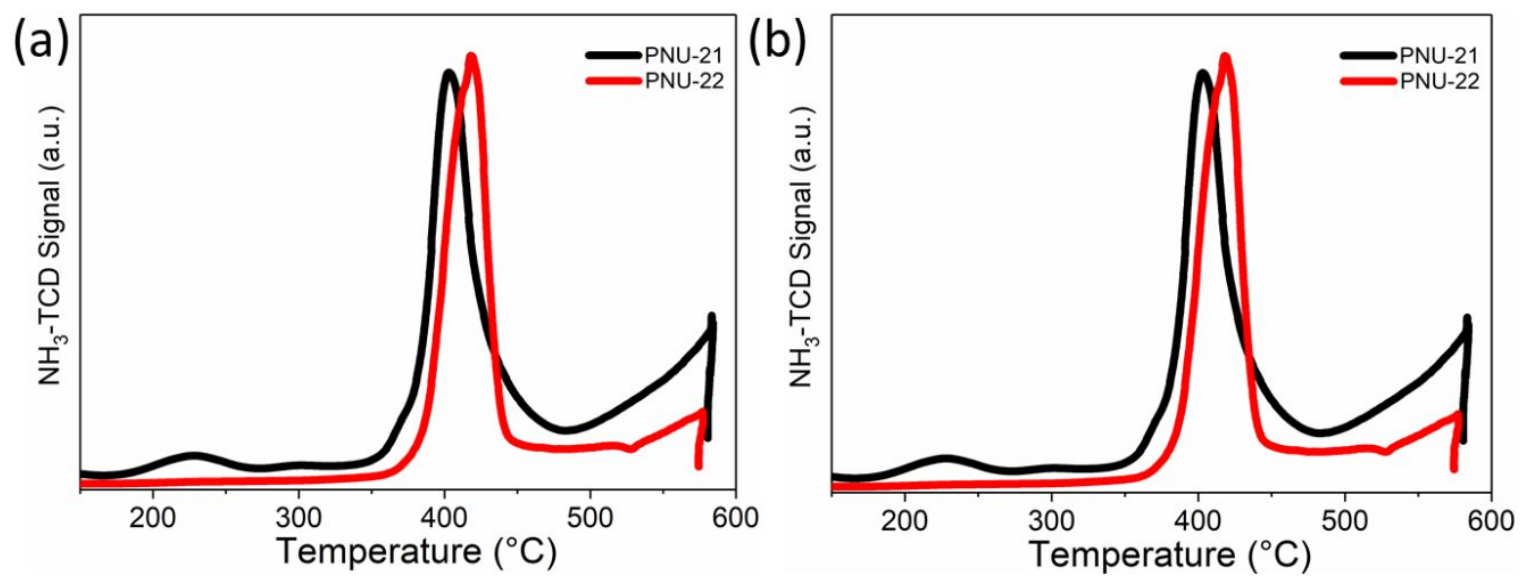

Figure S13. (a) $\mathrm{NH}_{3}-\mathrm{TPD}$ and (b) $\mathrm{CO}_{2}-\mathrm{TPD}$ results of synthesized catalysts.
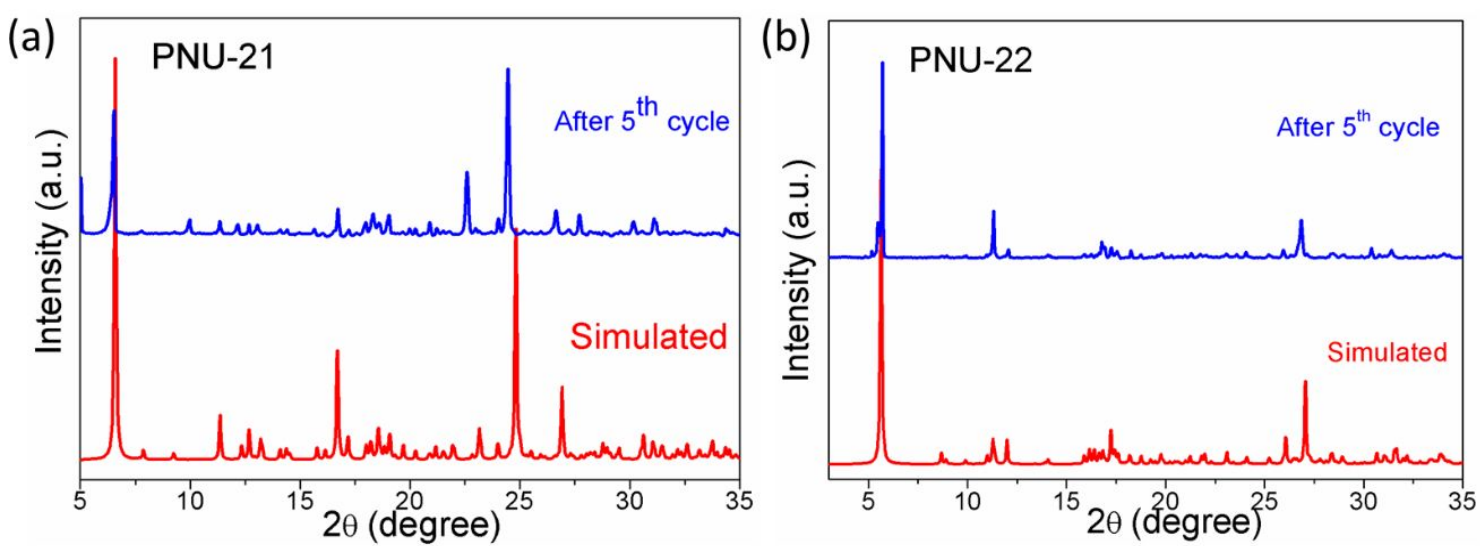

Figure S14. PXRD patterns of reused catalysts confirming their structural integrity after third catalytic cycle. 


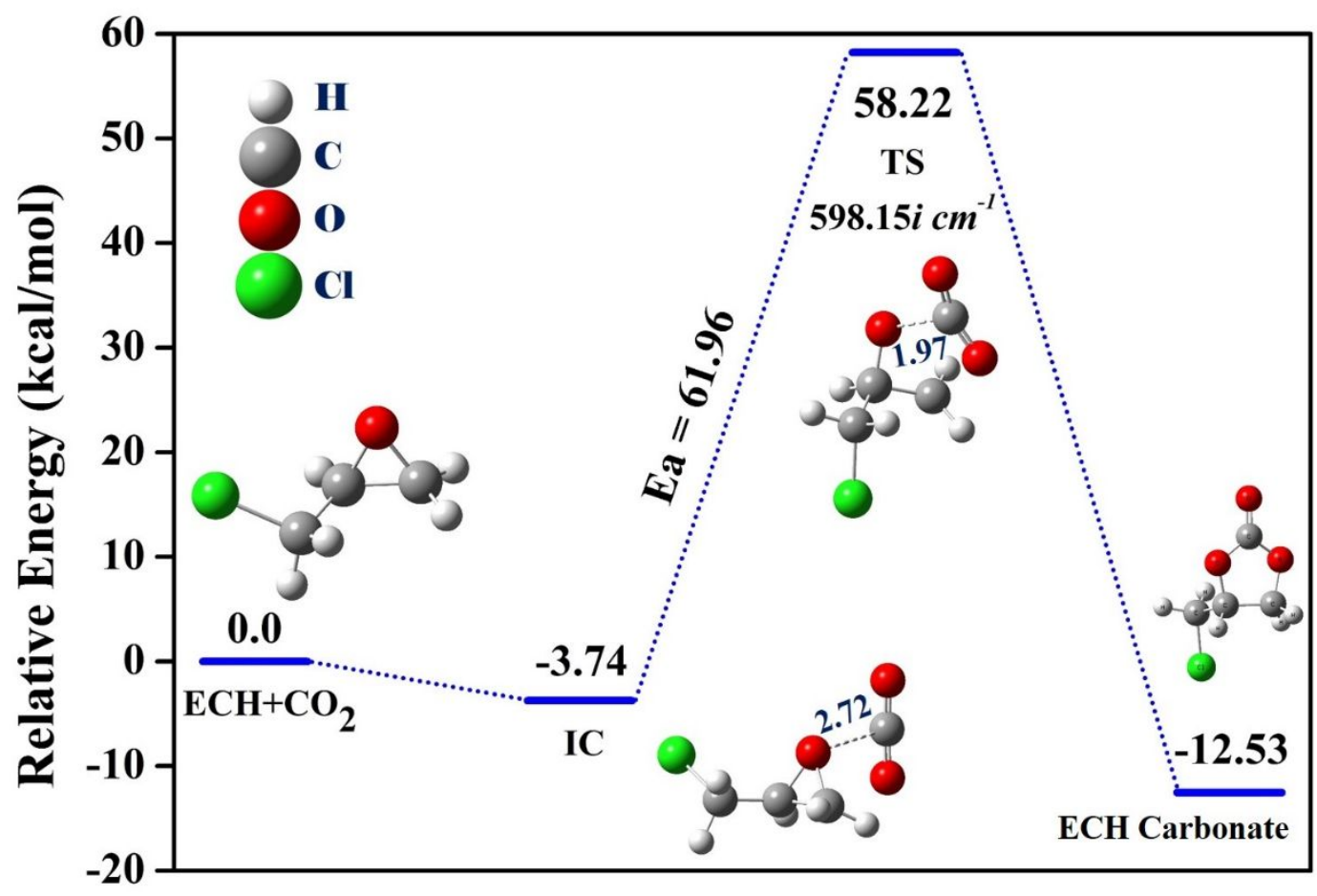

Figure S15. Relative free energy diagram of cycloaddition of $\mathrm{ECH}$ and $\mathrm{CO}_{2}$. The distances are given in $\AA$.

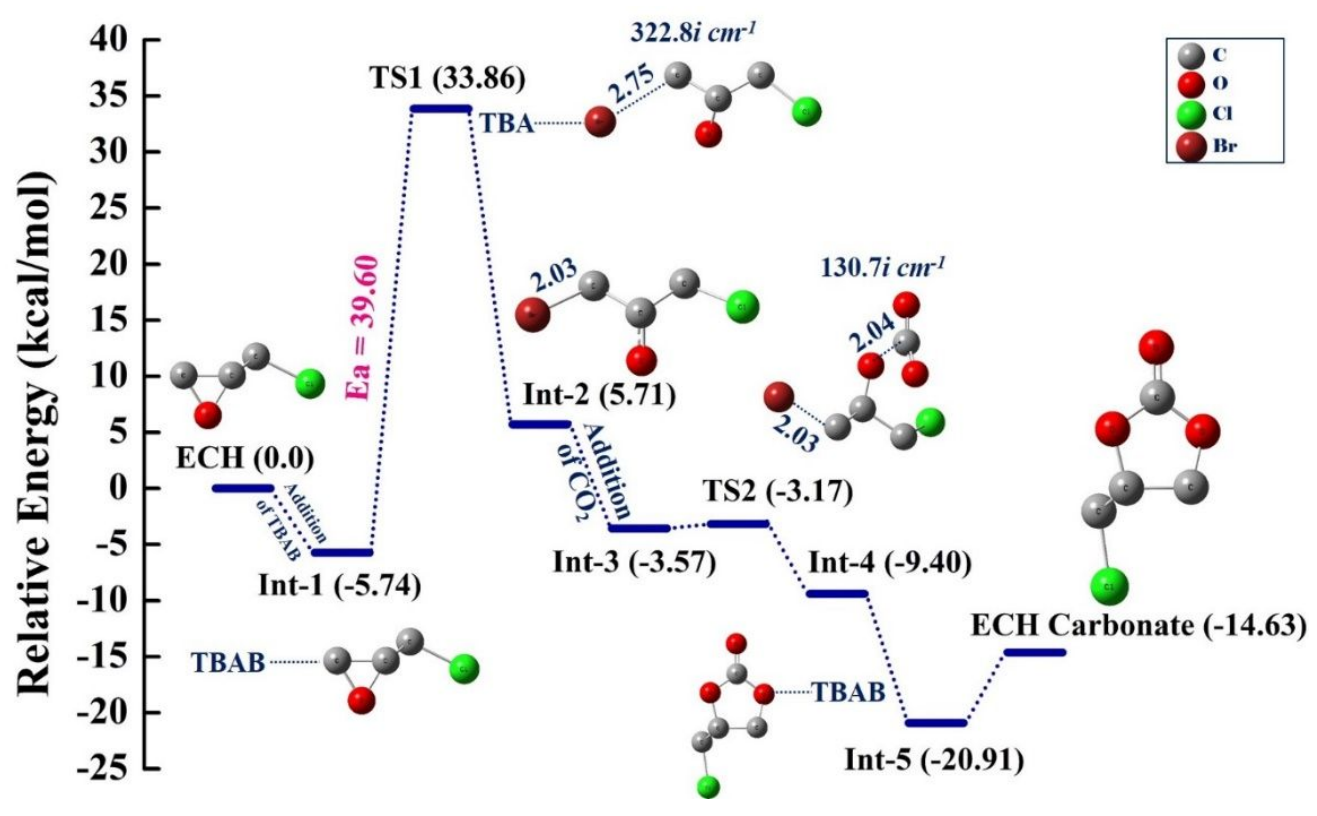

Figure S16. Relative free energy diagram of TBAB catalyzed cycloaddition of $\mathrm{ECH}$ and $\mathrm{CO}_{2}$. All the distances in the representative images of intermediates and transition states are in $\AA$. The hydrogen atoms are omitted here for clarity. 


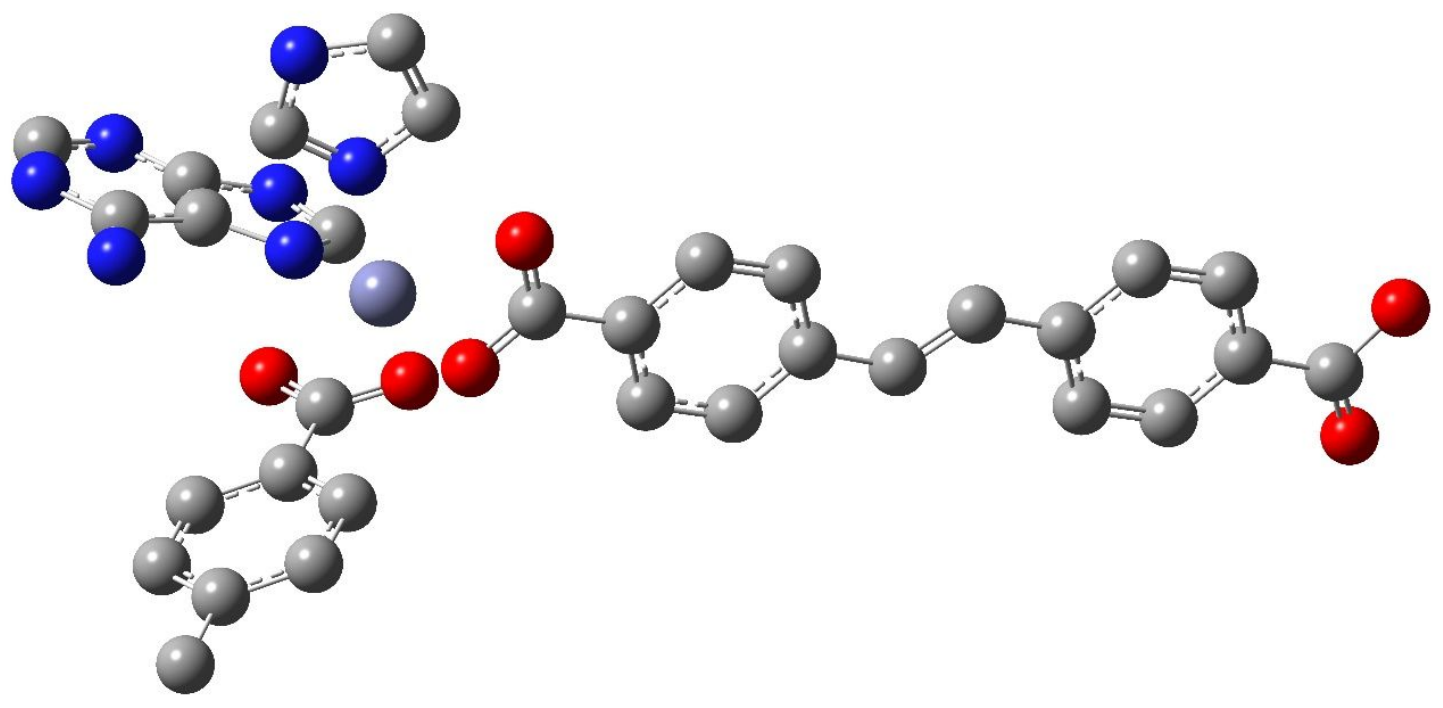

Figure S17. Optimized geometry of the modeled PNU-21. The hydrogen atoms are omitted here for clarity. 
Table S1. Crystal data and refinement parameters for PNU-21 and PNU-22.

\begin{tabular}{|c|c|c|}
\hline Identification code & PNU-21 & PNU-22 \\
\hline Chemical formula & $\mathrm{C}_{42} \mathrm{H}_{32} \mathrm{~N}_{10} \mathrm{O}_{9} \mathrm{Zn}_{2}$ & $\mathrm{C}_{42} \mathrm{H}_{30} \mathrm{~N}_{10} \mathrm{O}_{8} \mathrm{Cd} 2$ \\
\hline Formula weight & 951.52 & 1027.56 \\
\hline Crystal Colour & Pale Yellow & Pale Yellow \\
\hline Crystal Size (mm) & $0.16 \times 0.08 \times 0.02$ & $0.10 \times 0.04 \times 0.02$ \\
\hline Temperature (K) & $150(2)$ & $150(2)$ \\
\hline Crystal System & Triclinic & Triclinic \\
\hline Space Group & $\mathrm{P} \overline{1}$ & $\mathrm{P} \overline{1}$ \\
\hline $\mathrm{a}(\AA)$ & $10.8553(17)$ & $10.3168(14)$ \\
\hline $\mathrm{b}(\AA)$ & $13.216(2)$ & $11.3269(15)$ \\
\hline c $(\AA)$ & $14.954(2)$ & $16.691(2)$ \\
\hline$\alpha\left(^{\circ}\right)$ & $107.837(3)$ & $71.359(2)$ \\
\hline$\beta\left(^{\circ}\right)$ & $102.100(3)$ & $78.489(2)$ \\
\hline$\gamma\left({ }^{\circ}\right)$ & $108.098(3)$ & $75.820(2)$ \\
\hline $\mathrm{Z}$ & 2 & 2 \\
\hline $\mathrm{V}\left(\AA^{3}\right)$ & $1827.7(5)$ & 1776.2(4) \\
\hline Density $\left(\mathrm{Mg} / \mathrm{m}^{3}\right)$ & 1.729 & 1.921 \\
\hline Absorption Coefficient $\left(\mathrm{mm}^{-1}\right)$ & 1.391 & 1.274 \\
\hline $\mathrm{F}(000)$ & 972 & 1024 \\
\hline Reflections Collected & 14183 & 12718 \\
\hline Independent Reflections & 7097 & 6205 \\
\hline $\mathrm{R}_{\text {(int) }}$ & 0.0438 & 0.0381 \\
\hline Number of parameters & 581 & 561 \\
\hline $\mathrm{S}$ (Goodness of Fit) on $\mathrm{F}^{2}$ & 1.051 & 1.109 \\
\hline Final R1/wR2 [I>2 $\sigma(\mathrm{I})]$ & $0.0547 / 0.1080$ & $0.0490 / 0.1139$ \\
\hline Weighted R1/wR2 (all data) & $0.0802 / 0.1174$ & $0.0667 / 0.1269$ \\
\hline CCDC Numbers & 1895565 & 1895566 \\
\hline \multicolumn{3}{|c|}{$\mathrm{R}=\Sigma|| \mathrm{F}_{\mathrm{o}}|-| \mathrm{F}_{\mathrm{c}} \| / \Sigma\left|\mathrm{F}_{\mathrm{o}}\right| ; \mathrm{wR}=\left[\Sigma \mathrm{w}\left(\mathrm{F}_{\mathrm{o}}^{2}-\mathrm{F}_{\mathrm{c}}^{2}\right)^{2} / \Sigma \mathrm{w}\left(\mathrm{F}_{\mathrm{o}}^{2}\right)^{2}\right]^{1 / 2}$} \\
\hline
\end{tabular}


Table S2. Selected bond lengths and bond angles for PNU-21 and PNU-22.

\begin{tabular}{|c|c|c|c|}
\hline \multicolumn{4}{|c|}{ PNU-21 } \\
\hline $\mathrm{Zn}(2)-\mathrm{O}(6) \# 1$ & 1.992(3) & $\mathrm{O}(3)-\mathrm{C}(16)$ & $1.303(5)$ \\
\hline $\mathrm{Zn}(2)-\mathrm{N}(7)$ & $2.026(3)$ & $\mathrm{O}(3)-\mathrm{Zn}(1) \# 3$ & $1.967(3)$ \\
\hline $\mathrm{Zn}(2)-\mathrm{N}(2) \# 2$ & $2.085(3)$ & $\mathrm{O}(6)-\mathrm{Zn}(2) \# 1$ & 1.992(3) \\
\hline $\mathrm{Zn}(2)-\mathrm{O}(9)$ & $2.089(4)$ & $\mathrm{N}(2)-\mathrm{Zn}(2) \# 4$ & $2.085(3)$ \\
\hline $\mathrm{Zn}(2)-\mathrm{O}(5)$ & $2.186(3)$ & $\mathrm{N}(5)-\mathrm{H}(5 \mathrm{~A})$ & 0.8900 \\
\hline $\mathrm{Zn}(1)-\mathrm{O}(3) \# 3$ & $1.967(3)$ & $\mathrm{N}(5)-\mathrm{H}(5 \mathrm{~B})$ & 0.8900 \\
\hline $\mathrm{Zn}(1)-\mathrm{N}(1)$ & $2.002(3)$ & $\mathrm{N}(5)-\mathrm{H}(5 \mathrm{C})$ & 0.8900 \\
\hline $\mathrm{Zn}(1)-\mathrm{O}(2)$ & $2.011(3)$ & $\mathrm{N}(9)-\mathrm{H}(9 \mathrm{~A})$ & $0.88(5)$ \\
\hline $\mathrm{Zn}(1)-\mathrm{N}(6)$ & $2.033(3)$ & $\mathrm{N}(10)-\mathrm{H}(10 \mathrm{~A})$ & 0.8600 \\
\hline $\mathrm{O}(1)-\mathrm{C}(1)$ & $1.239(5)$ & $\mathrm{N}(10)-\mathrm{H}(10 \mathrm{~B})$ & 0.8600 \\
\hline $\mathrm{O}(2)-\mathrm{C}(1)$ & $1.290(5)$ & & \\
\hline $\mathrm{O}(6) \# 1-\mathrm{Zn}(2)-\mathrm{N}(7)$ & $118.70(12)$ & $\mathrm{N}(1)-\mathrm{Zn}(1)-\mathrm{N}(6)$ & $121.61(13)$ \\
\hline $\mathrm{O}(6) \# 1-\mathrm{Zn}(2)-\mathrm{N}(2) \# 2$ & $110.30(12)$ & $\mathrm{O}(2)-\mathrm{Zn}(1)-\mathrm{N}(6)$ & $96.15(13)$ \\
\hline $\mathrm{N}(7)-\mathrm{Zn}(2)-\mathrm{N}(2) \# 2$ & $130.34(13)$ & $\mathrm{C}(1)-\mathrm{O}(2)-\mathrm{Zn}(1)$ & $130.0(3)$ \\
\hline $\mathrm{O}(6) \# 1-\mathrm{Zn}(2)-\mathrm{O}(9)$ & $90.76(13)$ & $\mathrm{C}(16)-\mathrm{O}(3)-\mathrm{Zn}(1) \# 3$ & 114.2(3) \\
\hline $\mathrm{N}(7)-\mathrm{Zn}(2)-\mathrm{O}(9)$ & $87.20(14)$ & $\mathrm{C}(27)-\mathrm{O}(5)-\mathrm{Zn}(2)$ & 119.0(3) \\
\hline $\mathrm{N}(2) \# 2-\mathrm{Zn}(2)-\mathrm{O}(9)$ & $84.49(14)$ & $\mathrm{C}(27)-\mathrm{O}(6)-\mathrm{Zn}(2) \# 1$ & $123.5(3)$ \\
\hline $\mathrm{O}(6) \# 1-\mathrm{Zn}(2)-\mathrm{O}(5)$ & $90.07(11)$ & $\mathrm{Zn}(2)-\mathrm{O}(9)-\mathrm{H}(9 \mathrm{D})$ & $128(3)$ \\
\hline $\mathrm{N}(7)-\mathrm{Zn}(2)-\mathrm{O}(5)$ & $95.16(12)$ & $\mathrm{Zn}(2)-\mathrm{O}(9)-\mathrm{H}(9 \mathrm{C})$ & $122(4)$ \\
\hline $\mathrm{N}(2) \# 2-\mathrm{Zn}(2)-\mathrm{O}(5)$ & $92.27(12)$ & $\mathrm{C}(17)-\mathrm{N}(2)-\mathrm{Zn}(2) \# 4$ & $124.9(3)$ \\
\hline $\mathrm{O}(9)-\mathrm{Zn}(2)-\mathrm{O}(5)$ & $176.74(13)$ & $\mathrm{C}(21)-\mathrm{N}(2)-\mathrm{Zn}(2) \# 4$ & 131.1(3) \\
\hline $\mathrm{O}(3) \# 3-\mathrm{Zn}(1)-\mathrm{N}(1)$ & $116.53(13)$ & $\mathrm{C}(25)-\mathrm{N}(9)-\mathrm{H}(9 \mathrm{~A})$ & $119(3)$ \\
\hline $\mathrm{O}(3) \# 3-\mathrm{Zn}(1)-\mathrm{O}(2)$ & $100.55(12)$ & $\mathrm{C}(25)-\mathrm{N}(10)-\mathrm{H}(10 \mathrm{~A})$ & 120.0 \\
\hline $\mathrm{N}(1)-\mathrm{Zn}(1)-\mathrm{O}(2)$ & $109.33(13)$ & $\mathrm{C}(25)-\mathrm{N}(10)-\mathrm{H}(10 \mathrm{~B})$ & 120.0 \\
\hline $\mathrm{O}(3) \# 3-\mathrm{Zn}(1)-\mathrm{N}(6)$ & $108.57(12)$ & $\mathrm{H}(10 \mathrm{~A})-\mathrm{N}(10)-\mathrm{H}(10 \mathrm{~B})$ & 120.0 \\
\hline $\mathrm{O}(1)-\mathrm{C}(1)-\mathrm{O}(2)$ & $123.9(4)$ & $\mathrm{O}(1)-\mathrm{C}(1)-\mathrm{C}(2)$ & $119.6(4)$ \\
\hline
\end{tabular}




\begin{tabular}{|c|c|c|c|}
\hline \multicolumn{5}{|c|}{ PNU-22 } \\
\hline $\mathrm{Cd}(1)-\mathrm{O}(2)$ & $2.149(4)$ & $\mathrm{N}(5)-\mathrm{H}(5 \mathrm{~B})$ & 0.8600 \\
\hline $\mathrm{Cd}(1)-\mathrm{N}(6)$ & $2.152(5)$ & $\mathrm{N}(7)-\mathrm{Cd}(2) \# 3$ & $2.277(5)$ \\
\hline $\mathrm{Cd}(1)-\mathrm{N}(1)$ & $2.228(5)$ & $\mathrm{N}(8)-\mathrm{H}(8 \mathrm{~A})$ & 0.8900 \\
\hline $\mathrm{Cd}(1)-\mathrm{O}(1) \# 1$ & $2.457(4)$ & $\mathrm{N}(8)-\mathrm{H}(8 \mathrm{~B})$ & 0.8900 \\
\hline $\mathrm{Cd}(1)-\mathrm{O}(5)$ & $2.564(4)$ & $\mathrm{N}(8)-\mathrm{H}(8 \mathrm{C})$ & 0.8900 \\
\hline $\mathrm{Cd}(2)-\mathrm{N}(2) \# 2$ & $2.205(5)$ & $\mathrm{O}(2)-\mathrm{Cd}(1)-\mathrm{N}(6)$ & $120.70(17)$ \\
\hline $\mathrm{Cd}(2)-\mathrm{O}(5)$ & $2.225(4)$ & $\mathrm{O}(2)-\mathrm{Cd}(1)-\mathrm{N}(1)$ & $108.21(17)$ \\
\hline $\mathrm{Cd}(2)-\mathrm{N}(7) \# 3$ & $2.277(5)$ & $\mathrm{N}(6)-\mathrm{Cd}(1)-\mathrm{N}(1)$ & $130.96(18)$ \\
\hline $\mathrm{Cd}(2)-\mathrm{O}(7) \# 4$ & $2.378(4)$ & $\mathrm{O}(2)-\mathrm{Cd}(1)-\mathrm{O}(1) \# 1$ & $91.16(15)$ \\
\hline $\mathrm{Cd}(2)-\mathrm{N}(10)$ & $2.546(5)$ & $\mathrm{N}(6)-\mathrm{Cd}(1)-\mathrm{O}(1) \# 1$ & $91.39(16)$ \\
\hline $\mathrm{O}(1)-\mathrm{C}(1)$ & $1.248(7)$ & $\mathrm{N}(1)-\mathrm{Cd}(1)-\mathrm{O}(1) \# 1$ & $90.88(16)$ \\
\hline $\mathrm{O}(1)-\mathrm{Cd}(1) \# 1$ & $2.457(4)$ & $\mathrm{O}(2)-\mathrm{Cd}(1)-\mathrm{O}(5)$ & $104.73(15)$ \\
\hline $\mathrm{O}(2)-\mathrm{C}(1)$ & $1.286(7)$ & $\mathrm{N}(6)-\mathrm{Cd}(1)-\mathrm{O}(5)$ & $85.92(16)$ \\
\hline $\mathrm{O}(3)-\mathrm{C}(16)$ & $1.216(8)$ & $\mathrm{N}(1)-\mathrm{Cd}(1)-\mathrm{O}(5)$ & $78.34(15)$ \\
\hline $\mathrm{O}(4)-\mathrm{C}(16)$ & $1.325(7)$ & $\mathrm{O}(1) \# 1-\mathrm{Cd}(1)-\mathrm{O}(5)$ & $162.91(13)$ \\
\hline $\mathrm{O}(4)-\mathrm{H}(4)$ & 0.8200 & $\mathrm{~N}(2) \# 2-\mathrm{Cd}(2)-\mathrm{O}(5)$ & $132.29(16)$ \\
\hline $\mathrm{O}(7)-\mathrm{Cd}(2) \# 4$ & $2.378(4)$ & $\mathrm{N}(2) \# 2-\mathrm{Cd}(2)-\mathrm{N}(7) \# 3$ & $120.31(17)$ \\
\hline $\mathrm{O}(8)-\mathrm{C}(32)$ & $1.252(7)$ & $\mathrm{O}(5)-\mathrm{Cd}(2)-\mathrm{N}(7) \# 3$ & $107.14(16)$ \\
\hline $\mathrm{N}(2)-\mathrm{Cd}(2) \# 2$ & $2.205(5)$ & $\mathrm{N}(2) \# 2-\mathrm{Cd}(2)-\mathrm{O}(7) \# 4$ & $102.50(16)$ \\
\hline $\mathrm{N}(5)-\mathrm{H}(5 \mathrm{~A})$ & 0.8600 & $\mathrm{O}(5)-\mathrm{Cd}(2)-\mathrm{O}(7) \# 4$ & $89.22(15)$ \\
\hline $\mathrm{O}(1)-\mathrm{C}(1)-\mathrm{O}(2)$ & $124.0(6)$ & $\mathrm{N}(7) \# 3-\mathrm{Cd}(2)-\mathrm{O}(7) \# 4$ & $80.43(16)$ \\
\hline $\mathrm{O}(1)-\mathrm{C}(1)-\mathrm{C}(2)$ & $120.3(5)$ & $\mathrm{N}(2) \# 2-\mathrm{Cd}(2)-\mathrm{N}(10)$ & $92.12(16)$ \\
\hline $\mathrm{O}(2)-\mathrm{C}(1)-\mathrm{C}(2)$ & $115.7(5)$ & $\mathrm{O}(5)-\mathrm{Cd}(2)-\mathrm{N}(10)$ & $89.10(15)$ \\
\hline $\mathrm{O}(7) \# 4-\mathrm{Cd}(2)-\mathrm{N}(10)$ & $161.61(15)$ & $\mathrm{N}(7) \# 3-\mathrm{Cd}(2)-\mathrm{N}(10)$ & $82.59(16)$ \\
\hline $\mathrm{C}(1)-\mathrm{O}(1)-\mathrm{Cd}(1) \# 1$ & $122.3(4)$ & $\mathrm{O}(3)-\mathrm{C}(16)-\mathrm{O}(4)$ & $124.1(6)$ \\
\hline $\mathrm{C}(16)-\mathrm{O}(4)-\mathrm{H}(4)$ & 109.5 & & \\
\hline $\mathrm{Symmetry} \operatorname{transformations}: \# 1 .-\mathrm{x}+1,-\mathrm{y}+1,-\mathrm{Z}+2 ; \# 2 .-\mathrm{x},-\mathrm{y}+2,-\mathrm{z}+2 ; \# 3 .-\mathrm{x},-\mathrm{y}+1,-\mathrm{z}+2 ;$ \\
$\# 4-\mathrm{x}+1,-\mathrm{y}+2,-\mathrm{Z}+1$. & & & \\
\hline & & & \\
\hline
\end{tabular}


Table S3. Details of hydrogen bonding interactions observed in the structure of PNU-21 and PNU-22.

\begin{tabular}{|c|c|c|c|}
\hline $\mathbf{D}-\mathbf{H} \cdots \mathbf{A}$ & $\mathbf{d}(\mathbf{H} \cdots \mathbf{A})(\AA)$ & $\mathbf{d}(\mathbf{D} \cdots \mathbf{A})(\AA)$ & $\angle \mathrm{D}-\mathbf{H} \cdots \mathbf{A}\left({ }^{\circ}\right)$ \\
\hline \multicolumn{4}{|c|}{ PNU-21 } \\
\hline $1 \mathrm{~N} 5-\mathrm{H} 5 \mathrm{~A} \cdots \mathrm{O}$ - 1 & 2.29 & $2.784(5)$ & 115 \\
\hline $\mathrm{N} 5-\mathrm{H} 5 \mathrm{C} \cdots \mathrm{O} 2 \# 1$ & 1.91 & $2.790(5)$ & 173 \\
\hline N9-H9A $\cdots$ O8\#2 & $1.75(5)$ & $2.628(6)$ & $176(6)$ \\
\hline $\mathrm{O} 9-\mathrm{H} 9 \mathrm{C} \cdots \mathrm{O} 8 \# 3$ & $1.74(7)$ & $2.595(6)$ & $160(6)$ \\
\hline $\mathrm{N} 10-\mathrm{H} 10 \mathrm{~A} \cdots \mathrm{O} 7 \# 2$ & 1.90 & $2.727(6)$ & 162 \\
\hline $\mathrm{C} 24-\mathrm{H} 24 \cdots \mathrm{O} 4 \# 2$ & 2.49 & $3.157(6)$ & 129 \\
\hline $\mathrm{C} 33-\mathrm{H} 33 \cdots \mathrm{N} 5 \# 1$ & 2.62 & $3.366(6)$ & 137 \\
\hline \multicolumn{4}{|c|}{ Symmetry code: \#1. 1-x, -1-y, 1-z; 2. 1-x, 1-y, 2-z; 3. -1+x, -1+y, -1+z. } \\
\hline $\mathbf{D}-\mathbf{H} \cdots \mathbf{A}$ & $\mathbf{d}(\mathbf{H} \cdots \mathbf{A})(\AA)$ & $\mathbf{d}(\mathbf{D} \cdots \mathbf{A})(\AA)$ & $\angle \mathrm{D}-\mathbf{H} \cdots \mathbf{A}\left({ }^{\circ}\right)$ \\
\hline \multicolumn{4}{|c|}{ PNU-22 } \\
\hline O4-H4 $\cdots$ N9\#1 & 1.93 & $2.733(7)$ & 167 \\
\hline $\mathrm{N} 5-\mathrm{H} 5 \mathrm{~A} \cdots \mathrm{O} 7 \# 2$ & 2.00 & $2.820(7)$ & 158 \\
\hline $\mathrm{N} 8-\mathrm{H} 8 \mathrm{~A} \cdots \mathrm{O} 3 \# 3$ & 2.15 & $2.790(8)$ & 129 \\
\hline $\mathrm{N} 8-\mathrm{H} 8 \mathrm{~B} \cdots \mathrm{O} 7 \# 4$ & 2.39 & $3.264(7)$ & 166 \\
\hline $\mathrm{C} 38-\mathrm{H} 38 \cdots \mathrm{N} 3 \# 5$ & 2.59 & $3.382(8)$ & 144 \\
\hline \multicolumn{4}{|c|}{$\begin{array}{l}\text { Symmetry code: \#1. } 2+x, y,-1+z ; 2.2-x, 2-y, 1-z ; 3 .-2+x, y, 1+z ; 4 .-1+x,-1+y, \\
1+z ; 5 . x,-1+y, z .\end{array}$} \\
\hline
\end{tabular}


Table S4. Comparison study of TOF of PNU-21/PNU-22 with other previously reported MOFs.

\begin{tabular}{|c|c|c|c|c|}
\hline MOF & $\begin{array}{c}\text { Amount of TBAB } \\
(\mathbf{m o l} \%)\end{array}$ & $\mathbf{C O}_{\mathbf{2}} \mathbf{( M P a )}$ & TOF $\mathbf{h}^{-1} \mathbf{)}$ & Ref. \\
\hline MOF-5 & 2.5 & 0.4 & 10 & $\mathrm{~S} 1$ \\
\hline IRMOF-3 & 0.64 & 1.2 & 4 & $\mathrm{~S} 2$ \\
\hline Cr-MIL-101 & 0.62 & 0.8 & 3 & $\mathrm{~S} 3$ \\
\hline Hf-NU-1000 & 10 & 0.1 & 0.5 & $\mathrm{~S} 4$ \\
\hline MOF-205 & 0.6 & 1.2 & 6 & $\mathrm{~S} 5$ \\
\hline MMCF-2 & 7.2 & 0.1 & 15 & S6 \\
\hline MMPF-9 & 7.2 & 0.1 & 14 & S7 \\
\hline PNU-21 & $\mathbf{0 . 3}$ & $\mathbf{0 . 4}$ & $\mathbf{2 0}$ & Present study \\
\hline PNU-22 & $\mathbf{0 . 3}$ & $\mathbf{0 . 4}$ & $\mathbf{1 7 . 5}$ & Present study \\
\hline
\end{tabular}




\section{${ }^{1} \mathrm{H}$ NMR Details of Cyclic Carbonates}

\section{Epichlorohydrin Carbonate}

$\left(600 \mathrm{MHz}, \mathrm{CDCl}_{3}\right): \delta 5.02-4.97(\mathrm{~m}, 1 \mathrm{H}), 4.63-4.59(\mathrm{~m}, 1 \mathrm{H}), 4.42(\mathrm{dd}, \mathrm{J}=8.6,5.7 \mathrm{~Hz}, 1 \mathrm{H})$, $3.83-3.79(\mathrm{~m}, 1 \mathrm{H}), 3.74(\mathrm{dd}, \mathrm{J}=12.5,3.5 \mathrm{~Hz}, 1 \mathrm{H})$.

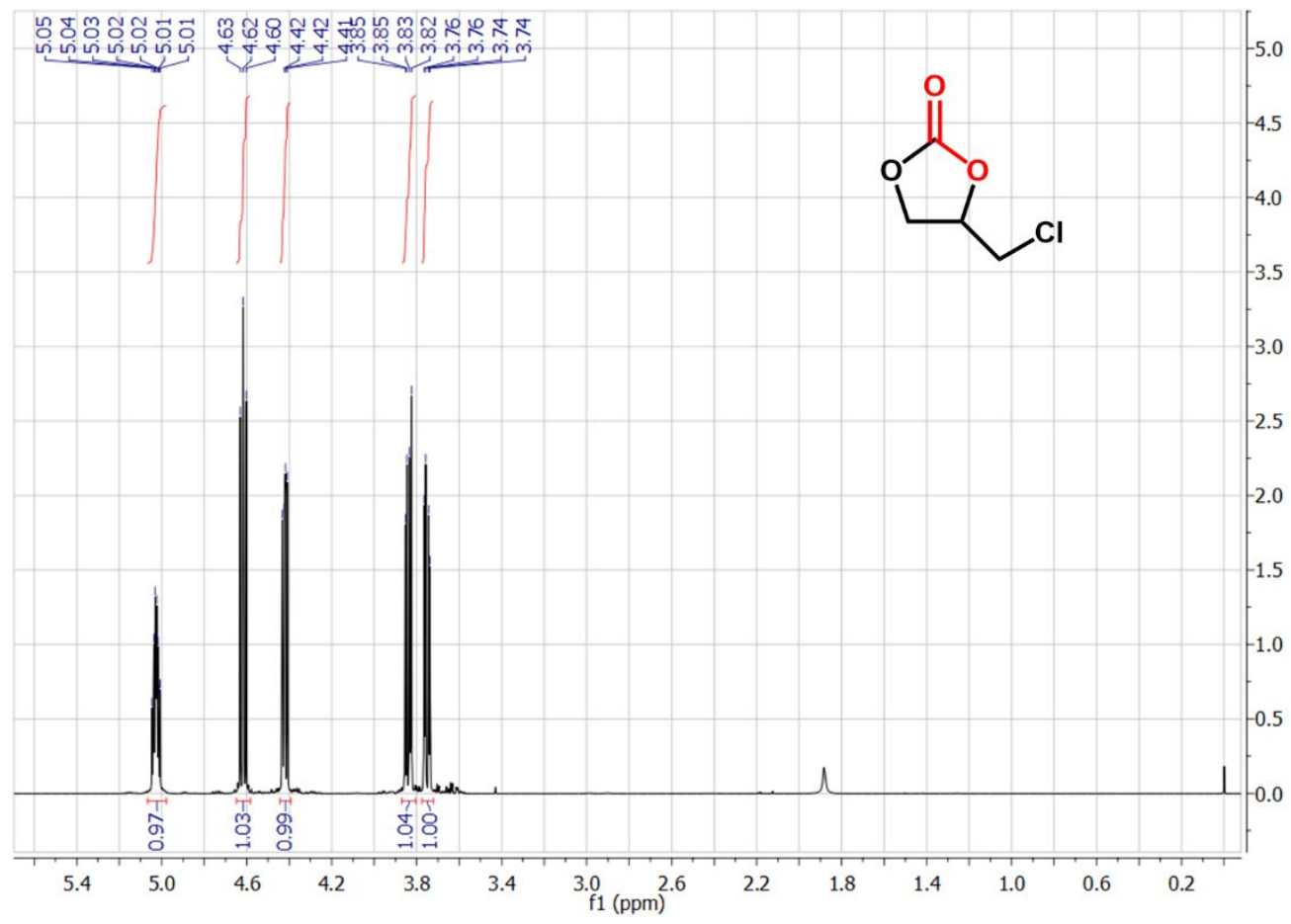




\section{Propylene Carbonates}

$\left(600 \mathrm{MHz}, \mathrm{CDCl}_{3}\right): \delta 4.94-4.87(\mathrm{~m}, 1 \mathrm{H}), 4.64-4.55(\mathrm{~m}, 1 \mathrm{H}), 4.06(\mathrm{dd}, \mathrm{J}=8.5,7.2 \mathrm{~Hz}, 1 \mathrm{H})$, $1.47(\mathrm{~d}, \mathrm{~J}=6.5 \mathrm{~Hz}, 3 \mathrm{H})$.

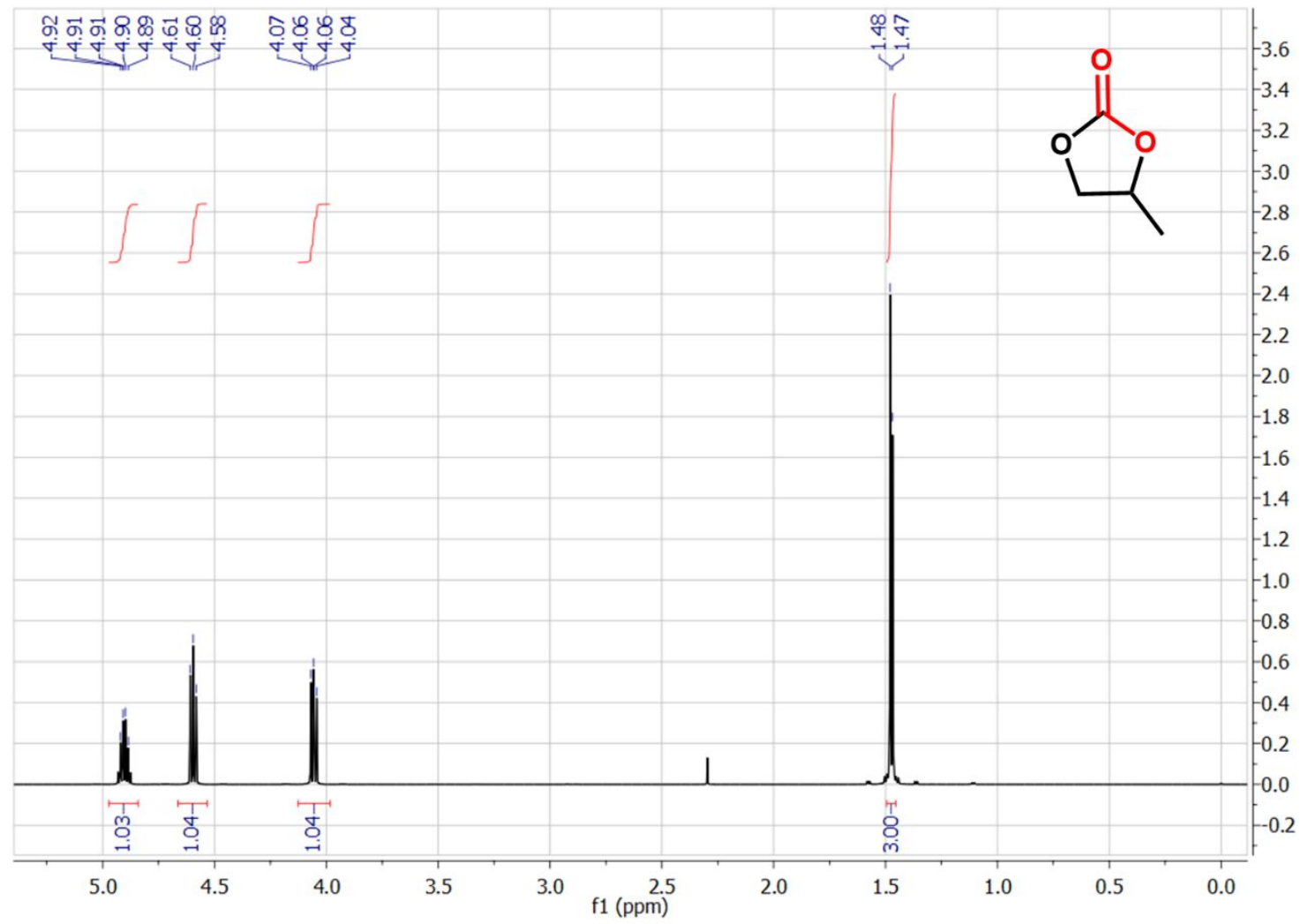




\section{Styrene Carbonate}

$\left(600 \mathrm{MHz}, \mathrm{CDCl}_{3}\right): \delta 7.40(\mathrm{dd}, 5 \mathrm{H}), 5.67(\mathrm{t}, \mathrm{J}=8.0 \mathrm{~Hz}, 1 \mathrm{H}), 4.80(\mathrm{t}, \mathrm{J}=8.5 \mathrm{~Hz}, 1 \mathrm{H}), 4.34(\mathrm{t}, \mathrm{J}=$ $8.1 \mathrm{~Hz}, 1 \mathrm{H})$.

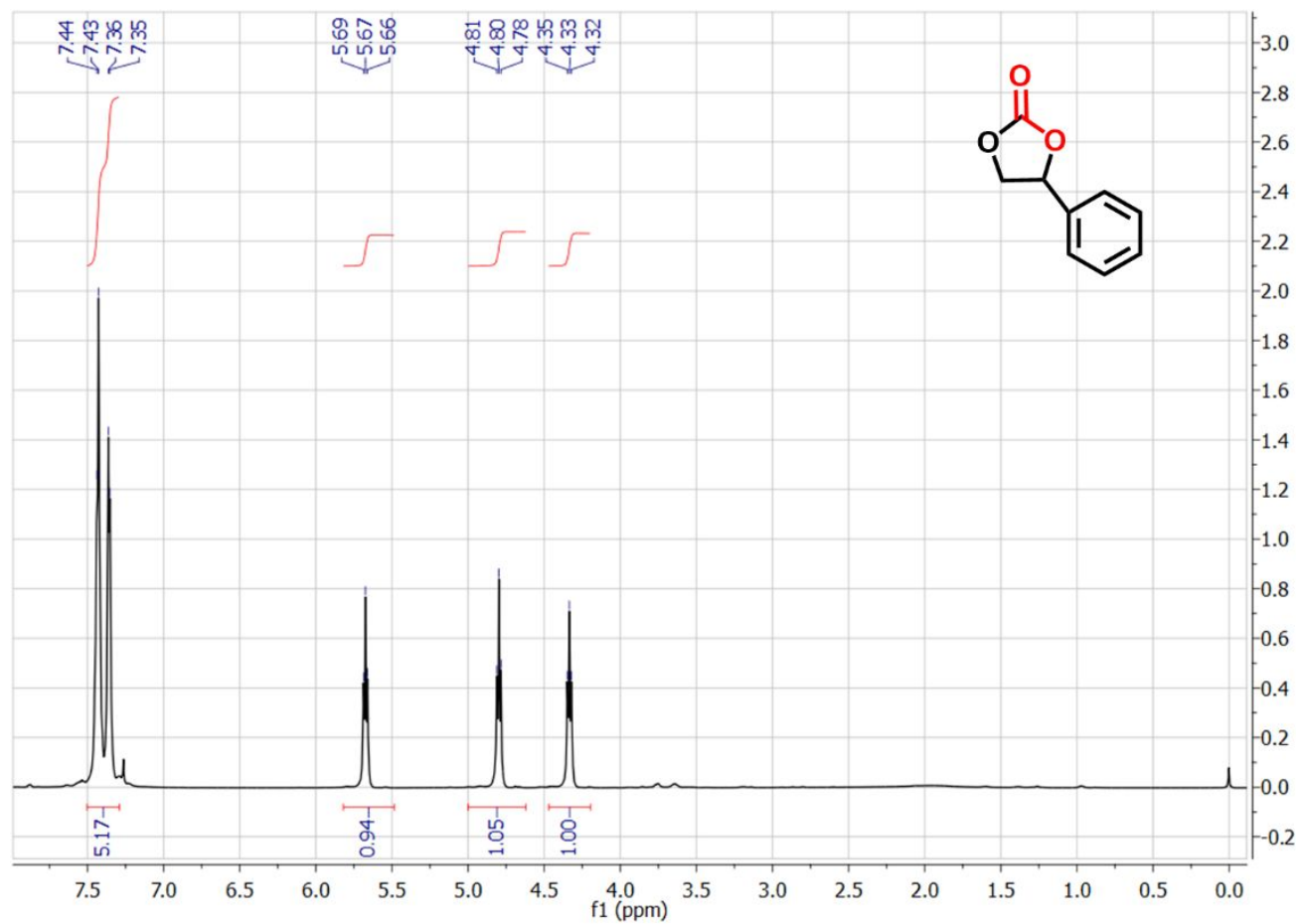




\section{Cyclohexene Carbonate}

$\left(600 \mathrm{MHz}, \mathrm{CDCl}_{3}\right): \delta=1.47(\mathrm{~m}, 2 \mathrm{H}), 1.64(\mathrm{~m}, 2 \mathrm{H}), 1.91(\mathrm{~m}, 4 \mathrm{H}), 4.72(\mathrm{~m}, 2 \mathrm{H})$

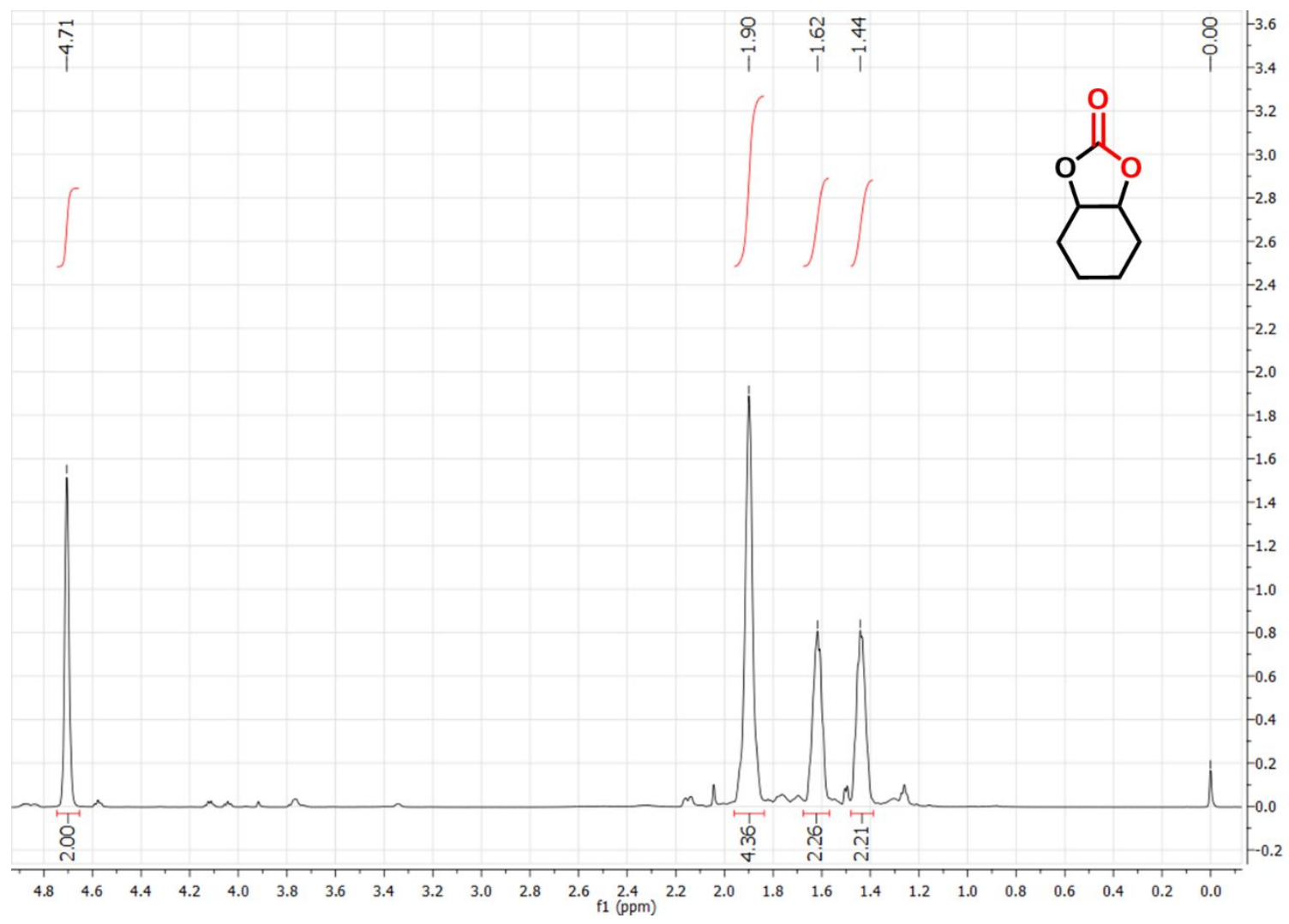

\section{Reference}

S1. Green Chem., 2009, 11, 1031-1036.

S2. RSC Adv., 2016, 6, 31153-31160.

S3. J. Catal., 2013, 298, 179-185.

S4. J. Am. Chem. Soc., 2014, 136, 15861-15864.

S5. ACS Appl. Mater. Interfaces, 2016, 8, 33723-33731.

S6. Angew. Chem., Int. Ed., 2014, 53, 2615-2619.

S7. Chem. Commun., 2014, 50, 5316-5318. 